PROCEEDINGS OF THE

AMERICAN MATHEMATICAL SOCIETY

Volume 134, Number 5, Pages 1405-1412

S 0002-9939(05)08116-5

Article electronically published on October 7, 2005

\title{
SUBADDITIVITY OF EIGENVALUE SUMS
}

\author{
MITSURU UCHIYAMA
}

(Communicated by Joseph A. Ball)

\begin{abstract}
Let $f(t)$ be a nonnegative concave function on $0 \leq t<\infty$ with $f(0)=0$, and let $X, Y$ be $n \times n$ matrices. Then it is known that $\|f(|X+Y|)\|_{1} \leq$ $\|f(|X|)\|_{1}+\|f(|Y|)\|_{1}$, where $\|\cdot\|_{1}$ is the trace norm. We extend this result to all unitarily invariant norms and prove some inequalities of eigenvalue sums.
\end{abstract}

\section{INTRODUCTION}

The eigenvalues of an $n \times n$ Hermitian matrix $H$ are denoted by $\lambda_{i}(H)(i=$ $1,2, \cdots, n)$ and arranged in increasing order, that is, $\lambda_{1}(H) \leq \lambda_{2}(H) \leq \cdots \leq$ $\lambda_{n}(H)$. The following sums are very important: for $1 \leq k \leq n$,

$$
\sigma_{(k)}(H):=\sum_{i=1}^{k} \lambda_{i}(H), \quad \sigma^{(k)}(H):=\sum_{i=n-k+1}^{n} \lambda_{i}(H) .
$$

These are represented as follows:

$$
\begin{aligned}
\sigma_{(k)}(H) & =\min \left\{\sum_{i=1}^{k}\left\langle H \mathbf{e}_{i}, \mathbf{e}_{i}\right\rangle:\left\{\mathbf{e}_{1}, \cdots, \mathbf{e}_{k}\right\} \text { is orthonormal }\right\}, \\
\sigma^{(k)}(H) & =\max \left\{\sum_{i=1}^{k}\left\langle H \mathbf{e}_{i}, \mathbf{e}_{i}\right\rangle:\left\{\mathbf{e}_{1}, \cdots, \mathbf{e}_{k}\right\} \text { is orthonormal }\right\} .
\end{aligned}
$$

Hence $\sigma_{(k)}(H)$ (or $\sigma^{(k)}(H)$ ) is a concave (or convex) function of $H$ (cf. 7]).

A norm $\|\cdot\|$ on the $n \times n$ matrices is called a unitarily invariant norm if

$$
\|U X V\|=\|X\|
$$

for all $X$ and for all unitary matrices $U$ and $V$. The operator norm $\|X\|$, Schatten p-norms

$$
\|X\|_{p}:=\left\{\sum_{i=1}^{n} \lambda_{i}(|X|)^{p}\right\}^{1 / p} \quad(p \geq 1),
$$

where $|X|=\left(X^{*} X\right)^{1 / 2}$, and Ky Fan $k$-norms $\|X\|_{(k)}:=\sigma^{(k)}(|X|)(k=1,2, \cdots, n)$ are typical unitarily invariant norms. It is well known that $\sigma^{(k)}(|X|) \leq \sigma^{(k)}(|Y|)$ $(k=1,2, \cdots, n)$ implies $\|X\| \leq\|Y\|$ for every unitarily invariant norm $\|\cdot\|$.

Received by the editors October 23, 2004 and, in revised form, December 11, 2004.

2000 Mathematics Subject Classification. Primary 47A30, 15A42; Secondary 47A75.

Key words and phrases. Trace, unitarily invariant norm, operator convex function. 
$\sigma^{(k)}(|X|) \leq \sigma^{(k)}(|Y|)(k=1,2, \cdots, n)$ means that the sequence $\left\{\lambda_{k}(|X|)\right\}_{k=1}^{n}$ is submajorized by $\left\{\lambda_{k}(|Y|)\right\}_{k=1}^{n}$ by definition; so one can restate some of the results in this paper by using the word "submajorized".

Let $f(t)$ be a nonnegative concave function on $0 \leq t<\infty$. Then Rotfel'd 8 and Thompson 9] (see also Theorem 4.2.14 of [4]) have shown that

$$
\|f(|X+Y|)\|_{1} \leq\|f(|X|)\|_{1}+\|f(|Y|)\|_{1} .
$$

We show in the fourth section that the above inequality holds for every unitarily invariant norm.

For Hermitian matrices $A$ and $B$ the inequality $A \leq B$ means $\langle A \mathbf{x}, \mathbf{x}\rangle \leq\langle B \mathbf{x}, \mathbf{x}\rangle$ for all vectors $\mathbf{x}$, where $\langle\cdot, \cdot\rangle$ is the inner product. A continuous function $\varphi$ defined on an interval $I$ is called an operator monotone function if for all $A, B$ whose eigenvalues lie in $I, A \leq B$ implies $\varphi(A) \leq \varphi(B)$. Likewise, a continuous function $\varphi$ on $I$ is called an operator convex function if

$$
\varphi(s A+(1-s) B) \leq s \varphi(A)+(1-s) \varphi(B)
$$

for all $A, B$ whose eigenvalues lie in $I$ and for every $s$ with $0 \leq s \leq 1 . \varphi$ is called an operator concave function if $-\varphi$ is operator convex.

We also give, in the third section, a simple proof of the following result [2]: for a nonnegative operator monotone function $\varphi$ on $[0, \infty)$ and for every unitarily invariant norm $\|\cdot\|$,

$$
\|\varphi(A+B)\| \leq\|\varphi(A)+\varphi(B)\| \quad(0 \leq A, B) .
$$

For details on this field we refer the readers to [4]. We appreciate the referee's useful comments.

\section{EsSEntial RESUlts}

For Hermitian matrices $A$ and $B$ the trace of $B A^{2} B-A B^{2} A$ vanishes. But, in general, it is difficult to estimate the trace of $C B A^{2} B C-C A B^{2} A C$. The next lemma follows from the more general result shown in [5]. However, this special case is useful and worth stating, so we prove it directly.

Lemma 2.1. Let $A \geq 0$ and $B \geq 0$, and let $Q$ be an orthogonal projection such that $Q B=B Q$. If $\inf \{\|B \mathbf{x}\|: Q \mathbf{x}=\mathbf{x},\|\mathbf{x}\|=1\} \geq \sup \{\|B \mathbf{x}\|:(1-Q) \mathbf{x}=\mathbf{x},\|\mathbf{x}\|=1\}$, then

$$
\begin{aligned}
\operatorname{tr} Q B A^{2} B Q & \geq \operatorname{tr} Q A B^{2} A Q \\
\operatorname{tr}(1-Q) B A^{2} B(1-Q) & \leq \operatorname{tr}(1-Q) A B^{2} A(1-Q) .
\end{aligned}
$$

Proof. Let

$$
A=\left[\begin{array}{cc}
A_{11} & A_{12} \\
A_{12}^{*} & A_{22}
\end{array}\right], \quad B=\left[\begin{array}{cc}
B_{1} & 0 \\
0 & B_{2}
\end{array}\right]
$$

be the decompositions of $A$ and $B$ corresponding to $\mathbf{C}^{n}=Q \mathbf{C}^{n} \oplus(1-Q) \mathbf{C}^{n}$. Then

$$
\begin{aligned}
Q B A^{2} B Q & =\left[\begin{array}{cc}
B_{1} A_{11}^{2} B_{1}+B_{1} A_{12} A_{12}^{*} B_{1} & 0 \\
0 & 0
\end{array}\right], \\
Q A B^{2} A Q & =\left[\begin{array}{cc}
A_{11} B_{1}^{2} A_{11}+A_{12} B_{2}^{2} A_{12}^{*} & 0 \\
0 & 0
\end{array}\right] .
\end{aligned}
$$


The assumption implies there are real numbers $m_{1}, m_{2} \geq 0$ such that $B_{1}^{2} \geq m_{1} \geq$ $m_{2} \geq B_{2}^{2}$. Since

$$
\begin{aligned}
\operatorname{tr} B_{1} A_{12} A_{12}^{*} B_{1} & =\operatorname{tr} A_{12}^{*} B_{1}^{2} A_{12} \geq m_{1} \cdot \operatorname{tr} A_{12}^{*} A_{12} \\
& \geq m_{2} \cdot \operatorname{tr} A_{12} A_{12}^{*} \geq \operatorname{tr} A_{12} B_{2}^{2} A_{12}^{*},
\end{aligned}
$$

we get (3). One can see (4) in the same way or by using (3).

Notice that $Q$ in the above lemma is the orthogonal projection onto the space spanned by eigenvectors of $B$ corresponding to $\lambda_{n}(B), \lambda_{n-1}(B), \cdots, \lambda_{n-k+1}(B)$ for some $k: 1 \leq k \leq n$. We can see that the above lemma is right for operators on an infinite Hilbert space as well if $A$ is of Hilbert-Schmidt class; so we used "inf (or sup)" instead of "min (or max)".

Corollary 2.2. Let $A \geq 0$ and $B \geq 0$, and let $Q$ be an orthogonal projection such that $Q B=B Q$. Suppose the sharper inequality:

$$
\inf \{\|B \mathbf{x}\|: Q \mathbf{x}=\mathbf{x},\|\mathbf{x}\|=1\}>\sup \{\|B \mathbf{x}\|:(1-Q) \mathbf{x}=\mathbf{x} \cdot\|\mathbf{x}\|=1\} .
$$

Then $\operatorname{tr} Q B A^{2} B Q=\operatorname{tr} Q A B^{2} A Q$ if and only if $Q A=A Q$.

Proof. Assume $\operatorname{tr} Q B A^{2} B Q=\operatorname{tr} Q A B^{2} A Q$. Then by (5), (6) we get

$$
\operatorname{tr} B_{1} A_{12} A_{12}^{*} B_{1}=\operatorname{tr} A_{12} B_{2}^{2} A_{12}^{*} \text {. }
$$

Since we can take $m_{1}$ and $m_{2}$ in the preceding proof as $m_{1}>m_{2}, \operatorname{tr} A_{12} A_{12}^{*}$ must vanish, and hence $A_{12}=0$. This implies $Q A=A Q$. The converse statement is clear.

In this paper the terms "increasing" and "decreasing" are used in the wider sense, that is, they mean "non-decreasing" and "non-increasing", respectively.

Proposition 2.3. Let $f(t)$ be a continuous function on $a \leq t \leq b$ with $a \geq 0$, and let $A, B$ be $n \times n$ nonnegative Hermitian matrices such that $a \leq A+B \leq b$. If $f(t)$ is decreasing and $t f(t)$ is increasing, or if $f(t)$ is increasing and $t f(t)$ is decreasing, then for $1 \leq k \leq n$

$$
\sigma^{(k)}\left(A^{1 / 2} f(A+B) A^{1 / 2}+B^{1 / 2} f(A+B) B^{\frac{1}{2}}\right) \geq \sigma^{(k)}((A+B) f(A+B)) .
$$

Hence, for any unitarily invariant norm $\|\cdot\|$

$$
\left\|A^{1 / 2} f(A+B) A^{1 / 2}+B^{1 / 2} f(A+B) B^{\frac{1}{2}}\right\| \geq\|(A+B) f(A+B)\| .
$$

Proof. The second inequality immediately follows from the first one, so we only show the first inequality. To do it we first assume that $f(t)$ is decreasing and $t f(t)$ is increasing. Notice that $f(t)$ is then nonnegative. Denote the unit eigenvector of $A+B$ corresponding to $\lambda_{i}:=\lambda_{i}(A+B)$ by $\mathbf{e}_{i} \quad(1 \leq i \leq n)$. As $(A+B) f(A+B) \mathbf{e}_{i}=$ $\lambda_{i} f\left(\lambda_{i}\right) \mathbf{e}_{i}$ and $\lambda_{i} f\left(\lambda_{i}\right) \leq \lambda_{i+1} f\left(\lambda_{i+1}\right)$, the less side of the first inequality equals $\sum_{i=n-k+1}^{n} \lambda_{i} f\left(\lambda_{i}\right)$. Therefore, by (2), it is sufficient to show

$$
\sum_{i=n-k+1}^{n}\left\langle\left(A^{1 / 2} f(A+B) A^{1 / 2}+B^{1 / 2} f(A+B) B^{1 / 2}\right) \mathbf{e}_{i} \cdot \mathbf{e}_{i}\right\rangle \geq \sum_{i=n-k+1}^{n} \lambda_{i} f\left(\lambda_{i}\right),
$$

which is equivalent to

$$
\operatorname{tr}\left\{P A^{1 / 2} f(A+B) A^{1 / 2} P+P B^{1 / 2} f(A+B) B^{1 / 2} P\right\} \geq \operatorname{tr} P(A+B) f(A+B) P,
$$


where $P$ is the orthogonal projection onto the space spanned by $\left\{\mathbf{e}_{n-k+1}, \cdots, \mathbf{e}_{n}\right\}$. Since $f\left(\lambda_{i}\right) \geq f\left(\lambda_{i+1}\right)$, by (4)

$$
\begin{aligned}
& \operatorname{tr} P A^{1 / 2} f(A+B) A^{1 / 2} P \geq \operatorname{tr} P f(A+B)^{1 / 2} A f(A+B)^{1 / 2} P, \\
& \operatorname{tr} P B^{1 / 2} f(A+B) B^{1 / 2} P \geq \operatorname{tr} P f(A+B)^{1 / 2} B f(A+B)^{1 / 2} P .
\end{aligned}
$$

Summing both inequalities yields the required inequality. We next assume that $f(t)$ is increasing and $t f(t)$ is decreasing; hence $f(t) \leq 0$. To see the required inequality we may prove

$$
\sigma_{(k)}\left(A^{1 / 2} f(A+B) A^{1 / 2}+B^{1 / 2} f(A+B) B^{1 / 2}\right) \leq \sigma_{(k)}((A+B) f(A+B)),
$$

because the traces of the matrices on both sides are identical. Since $t f(t)$ is decreasing, by (11) it is enough to show that

$$
\operatorname{tr}\left(P A^{1 / 2} f(A+B) A^{1 / 2} P+P B^{1 / 2} f(A+B) B^{1 / 2} P\right) \leq \sum_{i=n-k+1}^{n} \lambda_{i} f\left(\lambda_{i}\right),
$$

where $P$ is the orthogonal projection onto the space spanned by $\left\{\mathbf{e}_{n-k+1}, \cdots, \mathbf{e}_{n}\right\}$. We can obtain this inequality in the same way as above.

When we study the operator convexity, we often encounter a pair of matrices $X$ and $Y$ with $X^{*} X+Y^{*} Y=1$. But in this case, $X X^{*}+Y Y^{*}$ is not necessarily contractive; in fact, suppose $A+B$ is invertible for $A, B \geq 0$. Then $(A+B)^{-1 / 2} A(A+B)^{-1 / 2}+(A+B)^{-1 / 2} B(A+B)^{-1 / 2}=1$; but putting $f(t)=1 / t$ in the above proposition, we get $\sigma^{(k)}\left(A^{1 / 2}(A+B)^{-1} A^{1 / 2}+B^{1 / 2}(A+B)^{-1} B^{1 / 2}\right) \geq k$. Furthermore, we get

Corollary 2.4. Let $A$ and $B$ be nonnegative Hermitian matrices such that $A+B$ is invertible. Then the following are equivalent:

(i) $H:=A^{1 / 2}(A+B)^{-1} A^{1 / 2}+B^{1 / 2}(A+B)^{-1} B^{1 / 2} \leq 1$,

(ii) $H=1$,

(iii) $A B=B A$.

Proof. (i) $\Rightarrow$ (ii). As we mentioned above, we get $\sigma^{(k)}(H) \geq \sigma^{(k)}(1)$. Thus (i) implies $\sigma^{(k)}(H)=\sigma^{(k)}(1)=k$ for $1 \leq k \leq n$ and hence $H=1$.

(ii) $\Rightarrow$ (iii). Assume $\lambda_{n}(A+B) \geq \cdots \geq \lambda_{n-k+1}(A+B)>\lambda_{n-k}(A+B)$. Let $\mathbf{e}_{i}$ be the unit eigenvector of $A+B$ corresponding to $\lambda_{i}(A+B)$, and let $Q$ be the orthogonal projection onto the space spanned by $\mathbf{e}_{n}, \cdots, \mathbf{e}_{n-k+1}$. Then by Lemma 2.1

$$
\begin{aligned}
k & =\operatorname{tr} Q H Q=\operatorname{tr} Q A^{1 / 2}(A+B)^{-1} A^{1 / 2} Q+\operatorname{tr} Q B^{1 / 2}(A+B)^{-1} B^{1 / 2} Q \\
& \geq \operatorname{tr} Q(A+B)^{-1 / 2} A(A+B)^{-1 / 2} Q+\operatorname{tr} Q(A+B)^{-1 / 2} B(A+B)^{-1 / 2} Q \\
& =\operatorname{tr} Q=k,
\end{aligned}
$$

from which it follows that

$$
\operatorname{tr} Q A^{1 / 2}(A+B)^{-1} A^{1 / 2} Q=\operatorname{tr} Q(A+B)^{-1 / 2} A(A+B)^{-1 / 2} Q .
$$

Thus by Corollary 2.2 we obtain $A Q=Q A$. Therefore, one can see that $A$ commutes to every spectral projection of $A+B$. Thus $A B=B A$. Needless to say, (i) follows from (iii). 


\section{Operator CONCAVE FUNCTIONS}

Henceforth, we give some applications of Proposition 2.3 and assume every function is continuous. To start with, we give an another proof of the first statement of Ando and Zhan's theorem:

Theorem A ([2]). Let $\varphi(t)$ be a nonnegative operator monotone function on $[0, \infty)$. Then for $n \times n$ Hermitian matrices $A \geq 0$ and $B \geq 0$,

$$
\sigma^{(k)}(\varphi(A)+\varphi(B)) \geq \sigma^{(k)}(\varphi(A+B)) \quad(1 \leq k \leq n) .
$$

Let $\psi(t)$ be a strictly increasing function on $[0, \infty)$ with $\psi(0)=0$ and $\psi(\infty)=\infty$ such that the inverse function $\psi^{-1}(t)$ is operator monotone. Then

$$
\sigma^{(k)}(\psi(A)+\psi(B)) \leq \sigma^{(k)}(\psi(A+B)) \quad(1 \leq k \leq n) .
$$

To prove the first inequality we may assume that $A+B$ is invertible. Then, since $(A+B)^{-1 / 2} A^{1 / 2}$ is contractive, by Hansen and Pedersen's inequality [6] we have

$$
\begin{aligned}
\varphi(A) & =\varphi\left(A^{1 / 2}(A+B)^{-1 / 2}(A+B)(A+B)^{-1 / 2} A^{1 / 2}\right) \\
& \geq A^{1 / 2}(A+B)^{-1 / 2} \varphi(A+B)(A+B)^{-1 / 2} A^{1 / 2}, \\
\varphi(B) & \geq B^{1 / 2}(A+B)^{-1 / 2} \varphi(A+B)(A+B)^{-1 / 2} B^{1 / 2} .
\end{aligned}
$$

Since $\varphi(t)$ is increasing and $\varphi(t) / t$ is decreasing, by Proposition 2.3 we get

$$
\begin{aligned}
\sigma^{(k)}(\varphi(A)+\varphi(B)) & \geq \sigma^{(k)}\left(A^{1 / 2}(\varphi / t)(A+B) A^{1 / 2}+B^{1 / 2}(\varphi / t)(A+B) B^{1 / 2}\right) \\
& \geq \sigma^{(k)}(\varphi(A+B)) \quad(1 \leq k \leq n) .
\end{aligned}
$$

We can similarly prove the following extension of the first statement in the above theorem:

Let $\varphi(t)$ be an operator monotone function on $[0, \infty)$. Then for $A, B, C \geq 0$ and for $k=1, \cdots, n$,

$$
\sigma^{(k)}(\varphi(A+B+C)) \geq \sigma^{(k)}(\varphi(A)+\varphi(B)+\varphi(C)) .
$$

Recall that $\varphi(t)$ on $[0, \infty)$ is operator monotone if and only if $\varphi(t)$ is operator concave and $\varphi(\infty)>-\infty$ (cf. Proposition 3.5 of [10]). As $\varphi(t)$ in the preceding theorem is operator concave, it is natural to ask for a similar inequality related to an operator convex function.

Proposition 3.1. Let $\psi(t)$ be a non-constant, increasing operator convex function on $[0, \infty)$. Then $\psi(t)$ is strictly increasing and its inverse function $\psi^{-1}(t)$ is operator concave on $\left[\psi^{-1}(0), \infty\right)$.

Proof. Assume $\psi(0)=0$. Then it is known that $\psi(t) / t$ is operator monotone on $(0, \infty)$. Since the right-side limit of $\frac{\psi(t)}{t}$ at $t=0$ exists and is nonnegative, $\psi(t) / t$ has the natural extension that is nonnegative and operator monotone on $[0, \infty)$; we denote it by $\psi(t) / t$ again. Since $\psi(t) / t$ is increasing, $\psi(t)$ is strictly increasing. By Lemma 5 of [1] the inverse function of $\psi(t)=t(\psi(t) / t)$ is operator monotone and hence operator concave. Assume next $\psi(0) \neq 0$. For $\varphi(t):=\psi(t)-\psi(0)$ we get $\psi^{-1}(t)=\varphi^{-1}(t-\psi(0))$. Since $\varphi^{-1}$ is operator concave, so is $\psi^{-1}(t)$.

The converse of the previous proposition does not holds; for instance, $t^{1 / 3}$ is an operator concave function on $[0, \infty)$, but its inverse function $t^{3}$ is not operator convex. 
The following corollary can be shown by making use of the second inequality of Theorem A.

Corollary 3.2. Let $\psi(t)$ be a non-constant and increasing operator convex function on $[0, \infty)$ with $\psi(0)=0$. Then for $n \times n$ Hermitian matrices $A \geq 0$ and $B \geq 0$,

$$
\sigma^{(k)}(\psi(A)+\psi(B)) \leq \sigma^{(k)}(\psi(A+B)) \quad(1 \leq k \leq n) .
$$

\section{MAin RESUlts}

We notice that if $X$ and $Y$ are contractive such that $X^{*} X+Y^{*} Y=1$ and if the spectra of $A$ and $B$ are both in an interval $I$, then the spectrum of $X^{*} A X+Y^{*} B Y$ is in $I$ as well. A function is said to be monotone if it is increasing or decreasing.

Proposition 4.1. Let $f(t)$ be a concave function on an interval $I$, and let $A, B$ be $n \times n$ Hermitian matrices with the spectra in $I$. Then for $X, Y$ such that $X^{*} X+$ $Y^{*} Y=1$ and for $k=1,2, \cdots, n$,

$$
\sigma_{(k)}\left(f\left(X^{*} A X+Y^{*} B Y\right)\right) \geq \sigma_{(k)}\left(X^{*} f(A) X+Y^{*} f(B) Y\right) .
$$

Moreover, if $f(t)$ is monotone, then

$$
\lambda_{k}\left(f\left(X^{*} A X+Y^{*} B Y\right)\right) \geq \lambda_{k}\left(X^{*} f(A) X+Y^{*} f(B) Y\right) .
$$

Proof. Let $\left\{\lambda_{i}\right\}_{i=1}^{n}$ be the eigenvalues of $X^{*} A X+Y^{*} B Y$ so that $f\left(\lambda_{1}\right) \leq f\left(\lambda_{2}\right) \leq$ $\cdots \leq f\left(\lambda_{n}\right)$, and let $\left\{\mathbf{e}_{i}\right\}$ be the corresponding eigenvectors. Then the left side of (77) equals $f\left(\lambda_{1}\right)+\cdots+f\left(\lambda_{k}\right)$. By the concavity of $f$, we have

$$
\begin{aligned}
& \sum_{i=1}^{k}\left\langle\left(X^{*} f(A) X+Y^{*} f(B) Y\right) \mathbf{e}_{i}, \mathbf{e}_{i}\right\rangle \\
= & \sum_{i=1}^{k}\left\{\left\|X \mathbf{e}_{i}\right\|^{2}\left\langle f(A) \frac{X \mathbf{e}_{i}}{\left\|X \mathbf{e}_{i}\right\|}, \frac{X \mathbf{e}_{i}}{\left\|X \mathbf{e}_{i}\right\|}\right\rangle+\left\|Y \mathbf{e}_{i}\right\|^{2}\left\langle f(B) \frac{Y \mathbf{e}_{i}}{\left\|Y \mathbf{e}_{i}\right\|}, \frac{Y \mathbf{e}_{i}}{\left\|Y \mathbf{e}_{i}\right\|}\right\rangle\right\} \\
\leq & \sum_{i=1}^{k}\left\{\left\|X \mathbf{e}_{i}\right\|^{2} f\left(\left\langle A \frac{X \mathbf{e}_{i}}{\left\|X \mathbf{e}_{i}\right\|}, \frac{X \mathbf{e}_{i}}{\left\|X \mathbf{e}_{i}\right\|}\right\rangle\right)+\left\|Y \mathbf{e}_{i}\right\|^{2} f\left(\left\langle B \frac{Y \mathbf{e}_{i}}{\left\|Y \mathbf{e}_{i}\right\|}, \frac{Y \mathbf{e}_{i}}{\left\|Y \mathbf{e}_{i}\right\|}\right\rangle\right)\right\} \\
\leq & \sum_{i=1}^{k} f\left(\left\langle\left(X^{*} A X+Y^{*} B Y\right) \mathbf{e}_{i}, \mathbf{e}_{i}\right\rangle\right)=\sum_{i=1}^{k} f\left(\lambda_{i}\right) .
\end{aligned}
$$

Thus, by (11) we get (7).

If $f(t)$ is increasing, we can arrange eigenvalues $\left\{\lambda_{i}\right\}_{i=1}^{n}$ as $\lambda_{i} \leq \lambda_{i+1}$ and $f\left(\lambda_{i}\right) \leq$ $f\left(\lambda_{i+1}\right)$. For every unit vector $\mathbf{x}$ that is a linear combination of $\mathbf{e}_{1}, \cdots, \mathbf{e}_{k}$,

$$
\begin{aligned}
\left\langle\left(X^{*} f(A) X+Y^{*} f(B) Y\right) \mathbf{x}, \mathbf{x}\right\rangle & \leq f\left(\left\langle\left(X^{*} A X+Y^{*} B Y\right) \mathbf{x}, \mathbf{x}\right\rangle\right) \\
& \leq f\left(\lambda_{k}\right),
\end{aligned}
$$

for $\left\langle\left(X^{*} A X+Y^{*} B Y\right) \mathbf{x}, \mathbf{x}\right\rangle \leq \lambda_{k}$. From this, by the mini-max theorem, (8) follows. It can be similarly shown even if $f(t)$ is decreasing.

Corollary 4.2. Let $f(t)$ be a monotone and concave function on $I$. If $0 \in I$ and $f(0) \geq 0$, then for all $X$ such that $X^{*} X \leq 1$,

$$
\lambda_{k}\left(f\left(X^{*} A X\right)\right) \geq \lambda_{k}\left(X^{*} f(A) X\right) \quad(1 \leq k \leq n) .
$$

Proof. Put $B=0$ and $Y=\left(1-X^{*} X\right)^{1 / 2}$ in (8). Since $X^{*} f(A) X+Y^{*} f(0) Y \geq$ $X^{*} f(A) X$, we obtain the required inequality. 
Corollary 4.3. Let $g(t)$ be a convex function on $I$. Then for all $n \times n$ Hermitian matrices $A, B$ with the spectra in $I$ and for all $X, Y$ such that $X^{*} X+Y^{*} Y=1$,

$$
\sigma^{(k)}\left(g\left(X^{*} A X+Y^{*} B Y\right)\right) \leq \sigma^{(k)}\left(X^{*} g(A) X+Y^{*} g(B) Y\right) \quad(1 \leq k \leq n) .
$$

Moreover, if $g(t)$ is monotone, then

$$
\lambda_{k}\left(g\left(X^{*} A X+Y^{*} B Y\right)\right) \leq \lambda_{k}\left(X^{*} g(A) X+Y^{*} g(B) Y\right) \quad(1 \leq k \leq n) .
$$

Proof. Since $\lambda_{k}(-H)=-\lambda_{n-k+1}(H)$, by putting $f=-g$ in Proposition 4.1, we get this corollary.

Corollary 4.3 has been shown in [3] when $X$ and $Y$ are both real numbers. Now we are in position to show the following main theorem.

Theorem 4.4. Let $f(t)$ be a nonnegative, continuous and concave function on $[0, \infty)$. Then for all $n \times n$ (not necessarily Hermitian) matrices $A$ and $B$ and for $k=1, \cdots, n$

$$
\sigma^{(k)}(f(|A+B|)) \leq \sigma^{(k)}(f(|A|))+\sigma^{(k)}(f(|B|)) .
$$

Proof. Since $f(t)$ is nonnegative and concave on $[0, \infty), f(t)$ is increasing. Though the right-side limit of $(f(t)-f(0)) / t$ at $t=0$ is not necessarily finite, by considering $f(t+\epsilon)-f(\epsilon)$ instead of $f(t)$ for an arbitrary $\epsilon>0$, we may assume that $f(t)$ is right diffentiable at $t=0$ and $f(0)=0$. Then we can extend $f(t) / t$ continuously to $[0, \infty)$ and denote the extension by $f(t) / t$ again. Since $f(t)$ is nonnegative and concave, $f(t) / t$ is decreasing on $[0, \infty)$.

We first show (9) in the case $A \geq 0$ and $B \geq 0$. In this case there is no loss of generality in assuming that $A+B$ is invertible. Since $f(t)=t \cdot f(t) / t$, by Proposition 2.3 .

$$
\begin{aligned}
& \sigma^{(k)}(f(A+B)) \\
\leq & \sigma^{(k)}\left(A^{1 / 2}(A+B)^{-1} f(A+B) A^{1 / 2}+B^{1 / 2}(A+B)^{-1} f(A+B) B^{1 / 2}\right) \\
\leq & \sigma^{(k)}\left(A^{1 / 2}(A+B)^{-1 / 2} f(A+B)(A+B)^{-1 / 2} A^{1 / 2}\right) \\
+ & \sigma^{(k)}\left(B^{1 / 2}(A+B)^{-1 / 2} f(A+B)(A+B)^{-1 / 2} B^{1 / 2}\right) \\
\leq & \sigma^{(k)}(f(A))+\sigma^{(k)}(f(B)),
\end{aligned}
$$

where the second inequality is due to the subadditivity of $\sigma^{(k)}$ and the last inequality follows from Corollary 4.2 ,

We next consider general matrices $A$ and $B$. Then there are unitary matrices $U$ and $V$ such that

$$
|A+B| \leq U^{*}|A| U+V^{*}|B| V
$$

(see [9). Hence we have $\lambda_{k}(f(|A+B|)) \leq \lambda_{k}\left(f\left(U^{*}|A| U+V^{*}|B| V\right)\right.$ for $1 \leq k \leq n$. Thus from the result shown above it follows that

$$
\begin{aligned}
\sigma^{(k)}(f(|A+B|)) & \leq \sigma^{(k)}\left(f\left(U^{*}|A| U+V^{*}|B| Y\right)\right) \\
& \leq \sigma^{(k)}\left(f\left(U^{*}|A| U\right)\right)+\sigma^{(k)}\left(f\left(V^{*}|B| Y\right)\right) \\
& =\sigma^{(k)}\left(U^{*} f(|A|) U\right)+\sigma^{(k)}\left(V^{*} f(|B|) V\right) \\
& =\sigma^{(k)}(f(|A|))+\sigma^{(k)}(f(|B|)) .
\end{aligned}
$$


By considering unitarily invariant norm $\|\cdot\|$ instead of $\sigma^{(k)}$ in the above proof, we have

Corollary 4.5. Under the same condition as Theorem 4.4

$$
\begin{gathered}
\|f(|A+B|)\| \leq\|f(|A|)\|+\|f(|B|)\| . \\
\text { REFERENCES }
\end{gathered}
$$

[1] T. Ando, Comparison of norms $\|f(A)-f(B)\|$ and $\|f(|A-B|)\|$, Math. Z., 197 (1988), 403-409. MR0926848 (90a:47021)

[2] T. Ando, X. Zhan, Norm inequalities related to operator monotone functions, Math. Ann., 315 (1999), 771-780. MR1727183 (2000m:47008)

[3] J. S. Aujla, F. C. Silva, Weak majorization inequalities and convex functions, Linear Alg. App., 369 (2003), 217-233. MR1988488 (2004g:47021)

[4] R. Bhatia, Matrix Analysis, Springer-Verlag, 1997. MR1477662 (98i:15003)

[5] J. C. Bourin, Some inequalities for norms on matrices and operators, Linear Alg. Appl., 292 (1999), 139-154. MR1689308 (2000b:47022)

[6] F. Hansen, G. K. Pedersen, Jensen's inequality for operators and Löwner's theorem, Math. Ann., 258 (1982), 229-241. MR0649196 (83g:47020)

[7] E. Lieb, H. Siedentop, Convexity and concavity of eigenvalue sums, J. Statistical Physics, 63 (1991), 811-816. MR:1116035 (92j:15010)

[8] S. Y. Rotfel'd, Remarks on the singular numbers of a sum of completely continuous operators, Functional Anal. Appl., 1 (1967), 252-253.

[9] R. C. Thompson, Convex and concave functions of singular values of matrix sums, Pacific J. of Math., 66 (1976), 285-290. MR0435104 (55:8066)

[10] M. Uchiyama, Inverse functions of polynomials and orthogonal polynomials as operator monotone functions, Trans. Amer. Math. Soc., 355 (2004), 4111-4123. MR1990577(2004g:47024)

Department of Mathematics, Fukuoka University of Education, Munakata, Fukuoka, 811-4192, JAPAN

E-mail address: uchiyama@fukuoka-edu.ac.jp 